\section{Rotura de vena porta por pseudoquiste pancreático y hemorragia abdominal}

\section{Portal vein rupture secondary to a pancreatic pseudocyst}

Las complicaciones vasculares de la pancreatitis son entidades poco frecuentes pero importantes por su alta mortalidad $^{1}$. El pseudoquiste pancreático puede producir erosión de los vasos adyacentes, dando lugar a formación de pseudoaneurismas, trombosis venosa y hemorragias. La afectación venosa con formación de una fístula con el páncreas es una complicación muy rara ${ }^{2}$. Presentamos el caso de un paciente con pancreatitis crónica que presentó como complicación un pseudoquiste pancreático con rotura del mismo en la vena porta causando hemorragia con desenlace fatal.

Paciente varón de 48 años, con antecedentes de tabaquismo, alcoholismo y antrectomia por ulcus gastroduodenal y diagnosticado un año antes del ingreso de pancreatitis enólica leve con presencia de dos pseudoquistes pancreáticos, uno adyacente a la cabeza de páncreas (entre vena porta y tronco celíaco) y otro que se extendía por la fascia pararrenal izquierda.

El día de su ingreso presentó dolor abdominal y fiebre, observándose en la TC abdominal un aumento del tamaño de los pseudoquistes (aunque inferiores a $5 \mathrm{~cm}$ ), diagnósticándose de pancreatitis aguda por lo que se inició tratamiento antibiótico empírico con piperacilina/tazobactam.

En las siguientes $24 \mathrm{~h}$ presentó empeoramiento del dolor abdominal, sin fiebre, con episodio de hipotensión brusca (75/42 $\mathrm{mmHg})$, que no remontó tras aporte de hasta 3 litros de cristaloides. En el estudio de laboratorio destacó la presencia de coagulopatía leve (actividad de protrombina $43 \%$, INR 1,54; tiempo de cefalina 46 seg.) y plaquetopenia (plaquetas $32 \times 10 \mathrm{e} 3 / \mu \mathrm{L}$ ), manteniendo cifras normales de hemoglobina y hematocrito; alteración de la función renal (creatinina 2,20 mg/dl); elevación de lactato $(6,28 \mathrm{mmol} / \mathrm{L})$ y lipasa $3.395 \mathrm{UI} / \mathrm{L}$; siendo normales las enzimas hepáticas.

En una nueva TC abdominal (fig. 1) se observó un pseudoquiste en cabeza y cuerpo de páncreas de $7,4 \times 6,5 \mathrm{~cm}$

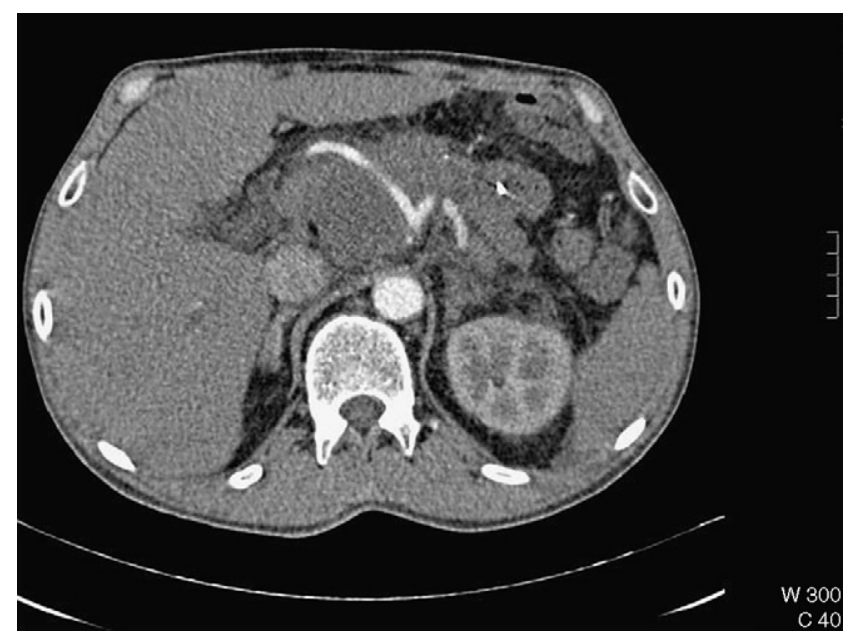

Figura 1 TC con contraste. Pseudoquiste pancreático. con contenido heterogéneo y alteración de la atenuación de la grasa mesentérica que rodeaba la lesión. El origen del tronco celíaco mostró un estrechamiento focal muy importante aunque con adecuado relleno de contraste de las arterias hepática, gastroduodenal y esplénica, además de un desplazamiento hacia la izquierda de la arteria hepática común y de la mesentérica superior, sin claros signos de extravasación activa de contraste.

Ante la sospecha de shock séptico de origen abdominal por sobreinfección de los pseudoquistes pancreáticos, el paciente se trasladó a la UCI presentando a su llegada los siguientes datos clínicos: TA $82 / 58 \mathrm{~mm} \mathrm{Hg}$, frecuencia cardíaca 99 lpm; $\mathrm{SatO}_{2}$ con gafas nasales a 3 litros del $96 \%$. Se realizó monitorización hemodinámica invasiva (Vigileo ${ }^{\circledR}$ ) obteniéndose un gasto cardiaco de $5 \mathrm{~L}$ y saturación venosa $\mathrm{O}_{2}$ de $76 \%$.

Tras 2 horas de ingreso en $\mathrm{UCl}$, comenzó de forma brusca con disnea intensa, desaturación arterial, taquicardia, escalofríos, livideces desde abdomen hacia ambos MMII, e hipotensión arterial. Se realizó intubación orotraqueal y ventilación mecánica $\left(\mathrm{FiO}_{2}\right.$ del $100 \%$, PEEP de 10$)$. En la Rx de tórax se observó patrón de distrés. Fue necesario administrar noradrenalina a dosis altas $(>1 \mathrm{mg} / \mathrm{dl})$, además de continuar con aporte de volumen. En el estudio de laboratorio se observó una acidosis metabólica mantenida y empeoramiento de la coagulopatía y plaquetopenia, sin anemización. Tras corrección de las alteraciones hematológicas se realizó laparotomía exploratoria que mostró un pseudoquiste gigante en cabeza de páncreas con salida de pus y ulceración del eje venoso de las venas mesentérica y esplénica en el origen de la vena porta. Ante la imposibilidad de reparación quirúrgica, el paciente falleció en quirófano.

La combinación de pancreatitis y hemorragia masiva en cavidad abdominal $(80,7 \%)$ o en tracto digestivo $(19,3 \%)$ es una complicación poco frecuente, pero potencialmente mortal. Este tipo de complicaciones están relacionadas con una enfermedad crónica en un 18\% (bien asociadas a pseudoquiste pancreático [63\%]) o a un pseudoaneurisma de un vaso principal) o con una pancreatitis aguda hasta en un $18 \%^{3}$. Los factores que aumentan el riesgo de hemorragia son la duración del proceso inflamatorio, la proximidad de los vasos a un pseudoquiste, la comunicación de este con el conducto pancreático o biliar y la oclusión de la vena esplécnica por trombosis ${ }^{4}$.

Los pseudoquistes pueden provocar erosión de los vasos adyacentes por diferentes mecanismos: compresión persistente, isquemia y destrucción de la pared vascular por la acción proteolítica de sus enzimas. Esto puede dar lugar, cuando afecta a arterias adyacentes, a la formación de pseudoaneurismas, hemorragia intraquística, o hemorragia libre intra- o retroperitoneal, mientras que cuando afecta a las venas, usualmente se desarrollan trombosis del sistema portal y sus ramas, con formación de varices (gástricas, colónicas y mesentéricas) ${ }^{3}$. La hemorragia es la complicación vascular más frecuente en la pancreatitis, siendo la fistula pancreático-portal una complicación extremadamente rara $^{5-9}$.

La presentación clínica de los pacientes que sufren una complicación vascular de la pancreatitis suele ser exacerbación repentina del dolor, distensión abdominal y síntomas clínicos de hemorragia, como hipotensión, taquicardia y taquipnea. La angiografía se considera la prueba más sen- 
sible para detectar la existencia y localización del punto sangrante, aunque la primera prueba diagnóstica suele ser la TC que permite identificar los pseudoaneurismas arteriales, la hemorragia dentro de un pseudoquiste y evaluar la morfología pancreática ${ }^{1,2,10}$. Diversos autores recomiendan realizar ecografía doppler en pacientes con pancreatitis crónica para el diagnóstico precoz de las posibles complicaciones vasculares ${ }^{1,2,10}$.

La intervención quirúrgica urgente es la opción terapéutica correcta cuando el paciente se encuentra hemodinámicamente inestable, pudiendo emplearse la embolización arterial en aquellos pacientes estables con evidencia angiográfica de hemorragia ${ }^{3}$. El pronóstico es mucho mejor en los pacientes con lesiones hemorrágicas situadas en el cuerpo y la cola de páncreas, ya que la mortalidad en estos pacientes ronda el $16 \%$, mientras que alcanza el $43 \%$ en los pacientes con lesión en cabeza pancreática ${ }^{4}$.

En el caso que presentamos, el pseudoquiste pancreático localizado en la cabeza de páncreas erosionó las paredes venosas produciendo la formación de un pseudoaneurisma venoso con posible paso de contenido del pseudoquiste al torrente circulatorio además de una importante hemorragia con desarrollo de shock mixto (séptico y hemorrágico) y fracaso multiorgánico. La localización de la rotura venosa hizo imposible su reparación en quirófano, produciéndose la muerte del paciente.

\section{Bibliografía}

1. Mallick IH, Winslet MC. Vascular complications of pancreatitis. J Pancreas. 2004;5:328-37.

2. Ko HS, Anders M, Dichl S, Dominguez E, Löhr M, Düber C. Portal vein erosion and acute abdominal hemorrhage as a complication of acute pancreatitis. Abdom Imaging. 2003;28:700-2.
3. Ferri RJ, Viciano V, Medrano J, Torro JA, Diago T, Garay M, et al. Seudoaneurisma esplénico, complicación del seudoquiste en la pancreatitis crónica. Cir Esp. 2000;68:507-9.

4. Nicolás de Prado I, Corral de la Calle MA, Nicolás de Prado JM, Sánchez Gallardo F, Medranda MA. Complicaciones vasculares de la pancreatitis. Rev Clin Esp. 2005;205:326-32.

5. De Perrot M, Berney T, Buhler L, Delgadillo X, Mentha G, Morel $\mathrm{P}$. Management of bleeding pseudoaneurysms in patients with pancreatitis. Br J Surg. 1999;86:29-32.

6. Takayama T, Kato K, Sano H, Katada N, Takeichi M. Spontaneous rupture of a pancreatic pseudocyst into the portal venous system. AJR Am J Roentgenol. 1986;147:935-6.

7. Van Steenbergen W, Ponette E. Pancreatico-portal fistula: a rare complication of chronic pancreatitis. Gastrointest Radiol. 1990;15:299-300.

8. Flati G, Andreu-Sandberg A, Sa Pinta M, Prorowska B, Carboni M. Potentially fatal bleeding in acute pancreatitis: pathophysiolgy, prevention, and treatment. Pancreas. 2003;26:8-14.

9. Packeisen J, Klingen D, Grezella F. Spontaneous rupture of a pancreatic pseudocyst into the portal vein. Zgastroenterl. 2001;39:961-4.

10. Vujic I. Vascular complications of pancreatitis. Radiol Clin North Am. 1989;27:81-91.

D. Díaz-Díaz*, A. Martínez de la Gándara, B. Bueno,

M. Villanova

y E. Palencia Herrejon

Unidad de Cuidados Intensivos, Hospital Universitario Infanta Leonor, Madrid, España

* Autor para correspondencia.

Correo electrónico: domingodiazdiaz@gmail.com

(D. Díaz-Díaz).

doi:10.1016/j.medin.2011.09.002

\section{Impactación antral y parada cardiorrespiratoria. Complicaciones del balón intragástrico}

\section{Antral impaction and cardiorespiratory arrest. Complications of the intragastric balloon}

La obesidad mórbida es una enfermedad crónica de tratamiento largo y, frecuentemente, frustrante. La colocación de un balón intragástrico constituye una intervención no quirúrgica, efectiva y de corta duración para perder peso como paso previo a la cirugía bariátrica, con intención de reducir el riesgo quirúrgico. Los pacientes con obesidad grado III o IV no candidatos a cirugía constituyen el otro gran grupo de pacientes subsidiarios de esta técnica de modo temporal, por periodos de entre 4 y 6 meses.

Las complicaciones asociadas suelen ser leves: náuseas, vómitos o reflujo gastroesofágico. Las complicaciones graves como perforación gástrica o esofágica, obstrucción intestinal o impactación son infrecuentes $(2,8-1,2 \%)$ y suelen resolverse con la retirada del dispositivo.
Presentamos el caso de una paciente que, tras ser tratada con un primer balón intragástrico durante 6 meses, recibe un segundo balón durante otros 2 meses, hasta la aparición de la complicación que describiremos a continuación.

\section{Caso clínico}

Mujer de 55 años que acude al Servicio de Urgencias del hospital por presentar en los 3 días previos un cuadro de vómitos e intolerancia a la ingesta y sin respuesta al tratamiento con antieméticos. Presenta episodio sincopal en su domicilio con recuperación espontánea y, tras escasos minutos en Urgencias, sufre parada cardiorrespiratoria que requiere 25 minutos de reanimación cardiopulmonar avanzada, incluyendo 5 desfibrilaciones.

De la primera analítica obtenida en Urgencias destaca sodio $131 \mathrm{mEq} / \mathrm{l}$, potasio $1,8 \mathrm{mEq} / \mathrm{l}$, creatinina $4,74 \mathrm{mg} / \mathrm{dl}$, urea $115 \mathrm{mg} / \mathrm{dl}$, pH 7,22 (venoso), bicarbonato $18,9 \mathrm{mEq} / \mathrm{l}$.

La paciente ingresa en la Unidad de Cuidados Intensivos ( $\mathrm{UCl}$ ) donde, tras colocación de sonda nasogástrica, se produce salida de $2.000 \mathrm{cc}$ de contenido gástrico. Tras 